Poincare Journal of Analysis E Applications

Vol. 2015 (2), Special Issue (IWWFA-II, Delhi), 105-114

CPoincare Publishers

\title{
WEIGHTED ABSOLUTE CONVERGENCE OF THE SERIES OF FOURIER-HAAR COEFFICIENTS
}

\author{
Boris Golubov ${ }^{\dagger}$ and Sergey Volosivets \\ Date of Receiving : : 25.03. 2015 \\ Date of Acceptance : $\quad 01.12 .2015$
}

\begin{abstract}
We formulate some sharp statements on absolute convergence of the series of Fourier-Haar coefficients. The two-dimensional analogs of some one-dimensional results are also formulated.
\end{abstract}

\section{Introduction}

The Haar orthonormal system $\left\{\chi_{n}\right\}_{n=1}^{\infty}$ had been constructed in 1909 (see [1]). By this system A. Haar gave positive answer on the question of D. Hilbert: is there an orthogonal system such that Fourier seies with respect to this system of any continuous function converges uniformly to that function.

Let us remind the definition of Haar system. We set $\chi_{1}(x) \equiv 1$ on $[0,1]$. After that we introduce the open dyadic intervals $I_{i}^{k}=\left((i-1) / 2^{k}, i / 2^{k}\right), i=1,2, \ldots, 2^{k}, k=0,1, \ldots$, and represent the natural number $n \geq 2$ in the form $n=2^{k}+i, i=1,2, \ldots, 2^{k}, k=0,1, \ldots$ Then we set $\chi_{n}(x)=2^{k / 2}$ for $x \in I_{2 i-1}^{k+1}, \chi_{n}(x)=-2^{k / 2}$ for $x \in I_{2 i}^{k+1}$ and $\chi_{n}(x)=0$ $x \in[0,1] \backslash \overline{I_{i}^{k}}$, where $\overline{I_{i}^{k}}$ is closure of the interval $I_{i}^{k}$. If the Haar function $\chi_{n}(x)$ has a jump in some point $x \in(0,1)$, then $\chi_{n}(x)=\left(\chi_{n}(x-0)+\chi_{n}(x+0)\right) / 2$. In the endpoints of the interval $[0,1]$ we set $\chi_{n}(0)=\chi_{n}(+0)$ and $\chi_{n}(1)=\chi_{n}(1-0)$. The Haar functions $\chi_{n}(x)$ are step functions.

The principal information on Fourier-Haar series may be found in the book [2].

For the function $f \in L_{p}[0,1], 1 \leq p<\infty$, we introduce integral modulus of continuity

$$
\omega(\delta, f)_{p}=\sup _{0 \leq h \leq \delta}\left(\int_{0}^{1-h}|f(t+h)-f(t)|^{p} d t\right)^{1 / p} \quad(0 \leq \delta \leq 1)
$$

2010 Mathematics Subject Classification. 42C10, 43C40, 41A17.

Key words and phrases. Haar system, Fourier-Haar coefficients, absolute convergence, function of bounded p-variation, best approximation, Nikoiskii type inequality .

The work of the first author is supported by the Russian Foundation for Basic Research under grant No. 14-01-00417. The work of the second author is supported by the Russian Foundation for Basic Research under grant No. 13-01-00238.

Communicated by. Sumit Kumar Sharma

${ }^{\dagger}$ Corresponding author. 